\title{
Rarefied Background Flow in a Vacuum Chamber Equipped with One-Sided Pumps
}

\author{
Chunpei Cai, ${ }^{*}$ Iain D. Boyd, ${ }^{\dagger}$ and Quanhua Sun ${ }^{\ddagger}$ \\ University of Michigan, Ann Arbor, Michigan 48109
}

\begin{abstract}
Spacecraft propulsion systems, such as Hall thrusters, are designed and tested in large vacuum chambers. The pumping capacity of modern facilities makes it possible to maintain pressures as low as $10^{-3}-10^{-4} \mathrm{~Pa}$ with thrusters in operation. For these vacuum chambers, a fundamental concern is the facility effects on the chamber performance. Several free molecular models are proposed to analyze the rarefied background flow inside a vacuum chamber equipped with one-sided vacuum pumps located at one chamber end. These models lead to various sets of analytical expressions linking several facility effects, such as pump sticking coefficient, pump area, and wall effects. These expressions can be used to estimate the pump sticking coefficient, to evaluate performance of vacuum chambers, and to aid constructing proper background flow for particle simulations. About 70 numerical simulations of background flows inside a vacuum chamber validate these models. These numerical simulations and analytical results indicate that these models are capable of predicting average background pressure and flow velocities. Depending on specific parameters, the background flow can have a significant nonzero mean velocity and cannot be considered to follow a Maxwellian velocity distribution.
\end{abstract}

\begin{tabular}{|c|c|}
\hline & Nomenclature \\
\hline$d$ & $=$ atomic diameter \\
\hline$f(C)$ & $=$ velocity distribution function \\
\hline$K n$ & $=$ Knudsen number \\
\hline$k$ & $=$ Boltzmann constant \\
\hline$m$ & $=$ atomic mass \\
\hline$\dot{m}$ & $=$ mass flow rate into chamber \\
\hline$n$ & $=$ number density \\
\hline$n_{\text {in }}$ & $=$ number density for flux into vacuum chamber \\
\hline$n_{X+}$ & $\begin{aligned}= & \text { number density for particles passing station } \\
& X \text { from one side }\end{aligned}$ \\
\hline$n_{X-}$ & $\begin{aligned}= & \text { number density for particles passing station } \\
& X \text { from other side }\end{aligned}$ \\
\hline$P_{b}$ & $=$ chamber backpressure \\
\hline$R$ & $=$ gas constant \\
\hline$S$ & $=$ surface area \\
\hline$S_{c}$ & $=$ chamber cross-sectional area \\
\hline$S_{p}$ & $=$ pump surface area \\
\hline$s$ & $=$ area ratio, $S_{p} / S_{c}$ \\
\hline$T_{p}$ & $=$ pump temperature \\
\hline$T_{\sim}$ & $=$ chamber wall temperature \\
\hline$\tilde{T}_{w p}$ & $=$ temperature ratio, $T_{w} / T_{p}$ \\
\hline$U$ & $=$ mean velocity \\
\hline$V$ & $=$ chamber volume \\
\hline$V_{p}$ & $\begin{aligned}= & \text { thermal velocity characterized by pump temperature, } \\
& {\left[2 k T_{p} /(\pi m)\right]^{1 / 2} }\end{aligned}$ \\
\hline$V_{w}$ & $\begin{aligned}= & \text { thermal velocity characterized by wall temperature, } \\
& {\left[2 k T_{w} /(\pi m)\right]^{1 / 2} }\end{aligned}$ \\
\hline$\alpha$ & $=$ pump sticking coefficient \\
\hline$\beta$ & $\begin{array}{l}=\text { particle transportation percentage from one chamber } \\
\text { end to other end }\end{array}$ \\
\hline
\end{tabular}

Received 27 July 2005; revision received 21 September 2005; accepted for publication 23 September 2005. Copyright (C) 2005 by Chunpei Cai. Published by the American Institute of Aeronautics and Astronautics, Inc., with permission. Copies of this paper may be made for personal or internal use, on condition that the copier pay the $\$ 10.00$ per-copy fee to the Copyright Clearance Center, Inc., 222 Rosewood Drive, Danvers, MA 01923; include the code 0887-8722/06 \$10.00 in correspondence with the CCC.

${ }^{*}$ Graduate Research Assistant, Department of Aerospace Engineering. Member AIAA.

$\dagger$ Professor, Department of Aerospace Engineering. Associate Fellow AIAA.

${ }^{\ddagger}$ Research Associate, Department of Aerospace Engineering. Senior Member AIAA.

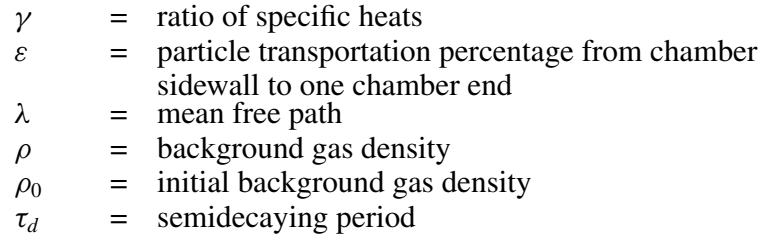

\section{Introduction}

$\mathbf{V}$ ACUUM chambers have wide applications for a variety of purposes such as materials processing and spacecraft electric propulsion experiments. The general goal of vacuum chambers is to maintain a low pressure. For example, in experiments testing a cluster of high-power electric plasma thrusters inside vacuum chambers, ${ }^{1-3}$ the backpressure was maintained at about $10^{-3}$ $10^{-4} \mathrm{~Pa}$. In such experiments, a high backpressure will distort the exhaust plume flow and affect the width of the ion energy distribution function through collisions between beam ions and neutral background particles. The presence of a high backpressure does not reflect the real situation in space and may adversely affect the experiments.

One primary concern regarding vacuum chambers is the facility effects on the chamber performance. There are several facility effects that have a significant impact on the vacuum chamber backpressure. ${ }^{4}$ The most significant effect is the pump sticking coefficient. The very low pressures obtained when testing electric thrusters are maintained using cryogenic pumps that employ gaseous helium. In such experiments, propellant frost will build up on cryopump surfaces and eventually will limit the pumping speed ${ }^{5}$; hence, even though the nominal sticking coefficient for gas-steel pump plates is high, in operation the pump sticking coefficient may be much lower. Vacuum pump area has an important effect as well. A typical vacuum chamber capable of testing the lifetime for electric plasma thrusters has a cylindrical shape with a minimum diameter of $6 \mathrm{~m}$ and a minimum length of $9 \mathrm{~m}$ (Ref. 4). For these long chambers, wall effects have an important influence on chamber performance.

In Ref. 1, a series of experiments was performed to test Hall thrusters in the Large Vacuum Test Facility (LVTF) at the University of Michigan. Different backpressures were measured with different numbers of pumps in operation and different mass flow rates from one or two thrusters. Several free molecular flow models have been proposed in Ref. 6 for analyzing these data and for studying the facility effects on this background flow. The general conclusions from these models and analysis are as follows: 
1) The pump sticking coefficient for the LVTF is close to 0.40 on average, though this value can vary within a small range under different operation conditions.

2) The background flow is composed of three groups of particles. The first group comes from the chamber end facing the thruster, the second group comes from the pump plate close to the other chamber end, and the last group comes from the end with a pump but not from pumps.

3) The velocities of the background flow do not follow a zerocentered Maxwellian distribution.

The pumps in the models of Ref. 6 are two-sided cyrogenic pumps where both sides of the pump are equally exposed to the background flows, and these models are essentially constant density models without detailed consideration of wall effects. The purposes of the present study are to investigate analytically and numerically the background flow inside a vacuum chamber equipped with onesided pumps at one chamber end. Compared with the models ${ }^{6}$ for two-sided pumps, one-sided pump models are simpler, hence, it is more convenient to investigate chamber sidewall effects in this case. Unlike the previous work in Ref. 6, which is based on available experimental measurements ${ }^{1}$ and three series of simulations, ${ }^{7}$ there are no data available for chambers equipped with one-sided pumps yet. Hence, the validity of the models in the present study will be compared with numerical simulations. In Ref. 8, the work regarding the facility effects for chambers equipped with one-sided pumps or two-sided pumps is summarized.

The rest of this paper is organized as follows: In Sec. II, the background and several general modeling assumptions are briefly reviewed; in Sec. III, three molecular flow models with discussions of results are presented; in Sec. IV, numerical simulations and comparisons with the models are presented; in Sec. V, the impact on numerical simulations are discussed; and the study is concluded in Sec. VI.

\section{Background and General Assumptions}

In Ref. 6, the background flow inside the LVTF, which is a vacuum chamber equipped with two-sided pumps that are located close to one chamber end, was studied. As stated earlier, the present study is focused on another type of chamber equipped with one-sided vacuum plates on one chamber end. The physical situation is quite similar to the background flow in the LVTF, hence, the conditions in the LVTF are briefly reviewed. The LVTF is used for testing plasma thrusters, and it is a cylindrical chamber with a diameter of $6 \mathrm{~m}$ and a length of $9 \mathrm{~m}$. Pumps are located on one chamber end, with seven cryopump plate surfaces. The pumps are maintained at an estimated temperature of $15 \mathrm{~K}$ using gaseous helium. When particles such as xenon atoms or ions hit the pumps, a fraction of the particles stick to the plates and the rest rebound diffusely with a thermal speed characterized by the pump temperature of $15 \mathrm{~K}$. A low-density plasma flow is exhausted from the thruster toward the other chamber end. Though there are ions in the plume flowfield, the ion number density is far lower than the neutral number density, and when these ions hit the chamber wall, they lose their charge and rebound diffusely as neutrals with a thermal speed characterized by the wall temperature of $300 \mathrm{~K}$. It is reasonable to assume that background neutrals move slowly from one chamber end toward the other end where the pumps are located.

Suppose that the gas flow is highly rarefied, it is reasonable to separate the chamber gas pressure into two parts: a universal background pressure and a plume pressure that only exists inside the plume. The study of the background pressure is the primary concern in this paper.

In the LVTF experiments, ${ }^{1-3}$ the backpressure of xenon is estimated using the ideal gas law $P_{b}=n k T_{w}$, where $n$ is the xenon number density measured using an ionization gauge and $T_{w}$ is the chamber temperature. In this study, the same formula is used to calculate the backpressure.

Several assumptions can be reasonable made based on the chamber operation conditions. First, pumps work effectively and create a low-density environment. This assumption results in a free molecular flow at the final steady state. With a typical final xenon backpressure of $10^{-3} \mathrm{~Pa}$ in the chamber, the mean free path of xenon atoms is about $2.86 \mathrm{~m}$. Second, the chamber wall temperature is $300 \mathrm{~K}$. Third, the background flow is one-dimensional. Fourth, the plume flow is neglected. The reflection of plume particles from one chamber end can be treated as entering neutral xenon gas through the chamber end with an area $S_{c}$ at the thruster mass flow rate $\dot{m}$ and wall temperature $T_{w}$. This end of the chamber is considered a source. Fifth, all pumps have the same sticking coefficient $\alpha$, the same pump temperature $T_{p}$, and a total pump area $S_{p}$. For the sake of simplicity, the total pump area $S_{p}$ is smaller than the chamber cross-sectional area $S_{c}$. When xenon atoms and ions hit the pump surfaces, by a probability of $\alpha$, they stick to the pumps and, by a probability of $1-\alpha$, they rebound with a thermal speed characterized by $T_{p}$. Hence, the pumps can be treated as a sink with a temperature $T_{p}$ and an area $S_{p}$ that is smaller than $S_{c}$. Because the flow is highly rarefied without many collisions between particles, the particles reflected from the pumps cannot hit the same pumps immediately without the necessary change of direction by collision with the other chamber end or the chamber sidewalls.

With the preceding assumptions, the background flow in the vacuum chamber can be simplified as one free molecular flow with two sources at both chamber ends and a sink for the pumps on or close to one chamber end.

In contrast with Ref. 6, in this study, the pumps are located on one chamber end and they are one-sided; hence, there is no postpump region and this greatly simplifies the analysis.

For any gas flow in equilibrium, the velocity distribution in any coordinate direction can be described as a full Maxwellian distribution. For a temperature $T$, the velocity distribution function for a stationary flow in one direction is

$$
f(C) \mathrm{d} C=[m /(2 \pi k T)]^{\frac{1}{2}} \exp \left[-(2 k T)^{-1} m C^{2}\right] \mathrm{d} C
$$

The mass flux in one direction across an area $S$ is

$$
\begin{aligned}
\dot{m}= & m n S \int_{0}^{\infty} C f(C) \mathrm{d} C \\
& =m n S \sqrt{(8 k T) /(\pi m)} / 4=m n_{+} S \sqrt{(2 k T) /(\pi m)}
\end{aligned}
$$

where $n_{+}=n / 2$ is the number density of particles moving in one direction.

Another important relation for this study is the number density after a group of particles reflect from a plate with a different temperature. Directly from Eq. (2), to preserve the flux, the following relation must hold:

$$
n_{1} \sqrt{T_{1}}=n_{2} \sqrt{T_{2}}
$$

where the subscripts 1 and 2 represent the incoming and reflected groups of particles, respectively. Equations (1-3) can be found in general textbooks on kinetic theory. ${ }^{9-11}$

\section{Free Molecular Flow Models}

\section{A. Model 1: From Mass Conservation Law}

Given a constant density distribution, the mass conservation law for the gas inside the vacuum chamber is

$$
\frac{\mathrm{d} \rho}{\mathrm{d} t}=\frac{\mathrm{d}\left(\int \rho \mathrm{d} v\right)}{\mathrm{d} t / V}=\frac{\left[\dot{m}-\alpha S_{p} \rho \sqrt{\left(8 k T_{w}\right) /(\pi m)} / 4\right]}{V}
$$

By the use of one initial condition, $\rho(t=0)=\rho_{0}$, and one steadystate condition, $\mathrm{d} \rho / \mathrm{d} t(t \rightarrow \infty)=0$, the solution for this equation can be obtained, which consists of one unsteady term and one steady term,

$$
\begin{aligned}
\rho(t) & =\left[\rho_{0}-(2 \pi m)^{\frac{1}{2}}\left(k T_{w}\right)^{-\frac{1}{2}} \dot{m}\left(\alpha S_{p}\right)^{-1}\right] \exp \left\{-\left[\alpha S_{p}\left(k T_{w}\right)^{\frac{1}{2}}\right.\right. \\
& \left.\left.\times(2 \pi m)^{-\frac{1}{2}} / V\right] t\right\}+(2 \pi m)^{\frac{1}{2}}\left(k T_{w}\right)^{-\frac{1}{2}} \dot{m}\left(\alpha S_{p}\right)^{-1}
\end{aligned}
$$


The mean velocity is

$$
U(t)=\dot{m}\left[S_{c} \rho(t)\right]^{-1}
$$

The pressure corresponding to the experimental measurements is

$$
\begin{aligned}
p_{b}(t) & =\left[\rho_{0} R T_{w}-\left(2 \pi R T_{w}\right)^{\frac{1}{2}} \dot{m}\left(\alpha S_{p}\right)^{-1}\right] \exp \left\{-\left[\alpha S_{p}\left(k T_{w}\right)^{\frac{1}{2}}\right.\right. \\
& \left.\left.\times(2 \pi m)^{-\frac{1}{2}} / V\right] t\right\}+\left(2 \pi R T_{w}\right)^{\frac{1}{2}} \dot{m}\left(\alpha S_{p}\right)^{-1}
\end{aligned}
$$

At steady state, the normalized pressure and the speed ratio are

$$
\begin{gathered}
P_{p} S_{c}\left(\dot{m} \sqrt{\gamma R T_{w}}\right)^{-1}=(2 \pi / \gamma)^{\frac{1}{2}} /(\alpha s) \\
U / \sqrt{2 R T_{w}}=\alpha s /(2 \sqrt{\pi})
\end{gathered}
$$

If the backpressure is known, then the pump sticking coefficient can be calculated using

$$
\alpha=\dot{m}\left(2 \pi R T_{w}\right)^{\frac{1}{2}}\left(P_{b} S_{p}\right)^{-1}
$$

This crude model, especially Eq. (7), relates many properties from the chamber, the pumps, the thruster, and the propellant, although the pump temperature is not included. Three conclusions can be drawn from this model:

1) It is evident from Eq. (7) that if the pumps work efficiently, the pressure will decrease and reach a final steady state. However, it also indicates that the unsteady term will take a finite time to decay significantly. For example, with the following LVTF parameters: $V=280 \mathrm{~m}^{3}, T_{w}=300 \mathrm{~K}, S_{p}=7.26 \mathrm{~m}^{2}$, and an assumption of $\alpha=0.40$, the decaying term is

$$
P_{b}(t)=C e^{-0.57 t}=C e^{-t / 1.75}
$$

The significant term in this expression is the semidecaying period $\tau_{d}=1.75 \mathrm{~s}$. In experiments, the pumps usually operate for at least several hours, and steady background flows are well established. However, in particle simulations of the rarefied plasma plume flow inside a vacuum chamber, usually it is modeled as an unsteady process with a time step of approximately $1 \times 10^{-7} \mathrm{~s}$. This requires over 50 million time steps for three semidecaying periods to reach a steady flow state. This presents a computational challenge to numerical simulations, and usually a full three-dimensional simulation of the whole chamber flow is too expensive.

2) The background gas flows toward the pump, and the highest speed is over $100 \mathrm{~m} / \mathrm{s}$ for xenon with the parameters $\alpha=s=1$.

3) No matter how efficiently the pumps work, there is a certain amount of finite backpressure in the vacuum chamber. This backpressure is represented by the second term of Eq. (7). The same expression also indicates that for a specific chamber with fixed parameters, at the final steady state, the background pressure is proportional to the mass flow rate from the thrusters. Although this is a crude approximation, experimental measurements ${ }^{1}$ support this conclusion. Reference 6 gives the details for the analysis of the experimental measurements of LVTF.

The background pressure can be calculated using Eq. (8) with a known sticking coefficient and a given mass flow rate. In general, for this model, the steady-state background pressure decreases as the sticking coefficient increases. At small values of $\alpha$, a $1 \%$ difference in the coefficient may result in a significant backpressure difference, whereas for large values the normalized pressure is not very sensitive to this parameter. For a numerical simulation of flows inside vacuum chambers, a correct sticking coefficient is critical.

\section{B. Model 2: From Kinetic Relations, Without Wall Effects}

In this model, a vacuum chamber is equipped with pumps on one end and only one side of the pumps is exposed to the background flow, as shown in Fig. 1. This model represents a practical and welldefined free molecular flow problem.

In the first step, model 2 still assumes a constant density distribution without strict treatment of wall effects, which is valid for a
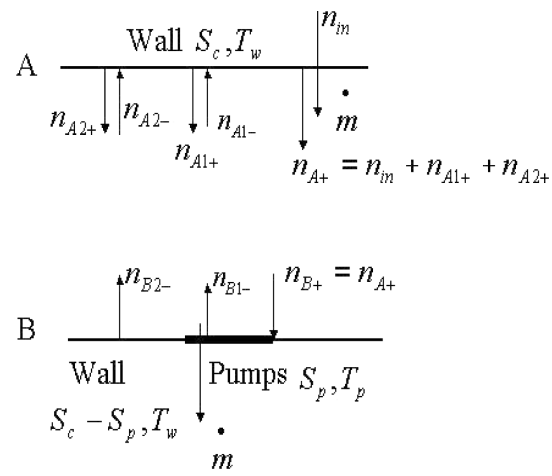

Fig. 1 Model 2.

short chamber assumption. In the next model, this assumption will be relaxed.

When the flow reaches a final steady state, the flux must be balanced everywhere along two directions, and this condition leads to several number density relations. Analysis of these flux and number density relations is the key step to analyze this problem.

At end A, a group of particles moves from A to B with a number density $n_{A+}=n_{\text {in }}+n_{A 1+}+n_{A 2+}$. At end $\mathrm{B}$, there are three number density relations from the flux relations:

1) Particles are reflected at end B but not from the pumps,

$$
n_{B 2-}=n_{A+}\left(1-S_{p} / S_{c}\right)=n_{A+}(1-s)
$$

2) For the mass flux relation, flux out of the chamber through the pumps at $\mathrm{B}$ equals the flux into the chamber at chamber end $\mathrm{A}$,

$$
\dot{m}=\alpha m n_{A+} S_{p} V_{w}=m n_{\text {in }} S_{c} V_{w}
$$

3) Particles are reflected from the pumps,

$n_{B 1-} \sqrt{T_{p}}=(1-\alpha) n_{A+}\left(S_{p} / S_{c}\right) \sqrt{T_{w}}=(1-\alpha) s n_{A+} \sqrt{T_{w}}$

The preceding relations yield the following expressions:

$$
\begin{gathered}
n_{A 1-}=n_{B 1-}=[(1-\alpha) / \alpha] \sqrt{T_{w} / T_{p}} n_{\text {in }} \\
n_{A 2+}=n_{A 2-}=n_{B 2-}=(1-s) /(\alpha s) n_{\text {in }} \\
n_{A 1+}=n_{B 1-} \sqrt{T_{p} / T_{w}}=[(1-\alpha) / \alpha] n_{\text {in }} \\
n_{A+}=n_{\text {in }} /(\alpha s)
\end{gathered}
$$

With the preceding relations, the full solutions for this model are

$$
\begin{aligned}
n_{A}= & n_{B}=n_{A+}+n_{B 1-}+n_{B 2-}=\left(\frac{2-s+s(1-\alpha) \sqrt{T_{w} / T_{p}}}{\alpha s}\right) n_{\mathrm{in}} \\
& =\frac{\dot{m}}{S_{c}} \sqrt{\frac{\pi}{2 k m T_{w}}}\left(\frac{2-s+s(1-\alpha) \sqrt{T_{w} / T_{P}}}{\alpha s}\right)
\end{aligned}
$$

$f(C)=$

$$
\left\{\begin{array}{l}
\frac{2 n_{\text {in }}}{\alpha s}\left(\frac{m}{2 \pi k T_{w}}\right)^{\frac{1}{2}} \exp \left(-\frac{m}{2 k T_{w}} C^{2}\right), \quad C>0 \\
\frac{2(1-\alpha) s \sqrt{T_{w} / T_{p}} n_{\text {in }}}{\alpha s}\left(\frac{m}{2 \pi k T_{p}}\right)^{\frac{1}{2}} \exp \left(-\frac{m}{2 k T_{p}} C^{2}\right) \\
+\frac{2(1-s) n_{\text {in }}}{\alpha s}\left(\frac{m}{2 \pi k T_{w}}\right)^{\frac{1}{2}} \exp \left(-\frac{m}{2 k T_{w}} C^{2}\right), \quad C<0
\end{array}\right.
$$


The normalized mean velocity, the pressure corresponding to experimental measurements $\left(P=n k T_{w}\right)$, and the pump sticking coefficients are as follows:

$$
\begin{gathered}
\frac{U}{\sqrt{2 R T_{w}}}=\frac{s \alpha}{\sqrt{\pi}\left(2-s+s(1-\alpha) \sqrt{T_{w} / T_{p}}\right)} \\
\frac{P_{b} S_{c}}{\dot{m} \sqrt{\gamma R T_{w}}}=\frac{n k T_{w} S_{c}}{\dot{m} \sqrt{\gamma R T_{w}}}=\sqrt{\frac{\pi}{2 \gamma}}\left(\frac{2-s+s(1-\alpha) \sqrt{T_{w} / T_{p}}}{\alpha s}\right) \\
\alpha=\frac{2-s+s \sqrt{T_{w} / T_{p}}}{s \sqrt{T_{w} / T_{p}}+P_{b} S_{P} /\left(\dot{m} \sqrt{\pi R T_{w} / 2}\right)}
\end{gathered}
$$

It is appropriate to discuss the range of validity for the preceding formulas. These expressions are based on a free molecular flow assumption; hence, for a specific mass flux and a specific propellant, it is not difficult to evaluate whether the assumption is correct. The number density $n_{A}$ from Eq. (19a) and the chamber end radius are selected as the characteristic quantities for the evaluation of the Knudsen number,

$$
\begin{aligned}
K n & =\frac{\lambda}{\sqrt{S_{C} / \pi}}=\frac{1}{\sqrt{2} \pi d^{2} \sqrt{S_{C}} n_{A}} \\
& =\frac{\alpha s m \sqrt{R T_{w} S_{C}}}{\pi d^{2} \dot{m}\left(2-s+(1-\alpha) s \sqrt{T_{w} / T_{p}}\right)}
\end{aligned}
$$

where the sticking coefficient in this formula should be computed with Eq. (20c) if not given as a known quantity.

Model 2 leads to exact solutions to a classical free molecular flow problem. Generally, a vacuum system consistent with model 2 works less efficiently than a vacuum chamber equipped with twosided pumps, which was studied in Ref. 6, because only the particles moving toward end $\mathrm{B}$ have a chance to be absorbed.

Relation (19b) indicates that there are three groups of particles moving inside the chamber. One group of particles travels toward the vacuum pump, whereas the other two groups travel from the pump direction, one of which is directly reflected from the pumps. Figure 2 shows several velocity distributions with different combinations of parameters. It is evident that the background flow cannot be described as a Maxwellian distribution, and the average velocity is not zero.

Figure 3 shows results obtained with Eq. (20b), which links the normalized average backpressure inside the chamber with the pump area and pump sticking coefficient. The temperature ratio is set to $T_{P} / T_{w}=15 / 300$. An increase in the pump area or pump sticking coefficient results in a lower background pressure. Figure 4 shows

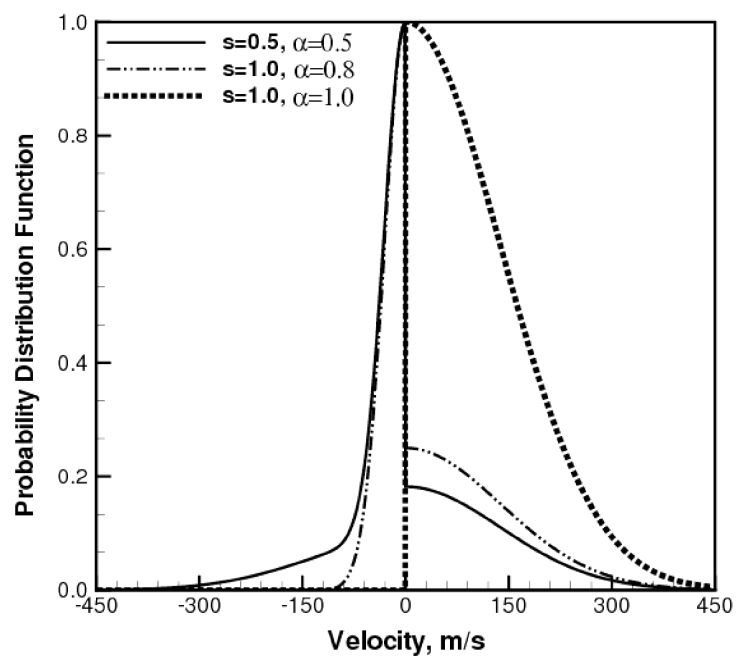

Fig. 2 Velocity distribution examples from Eq. (19b).

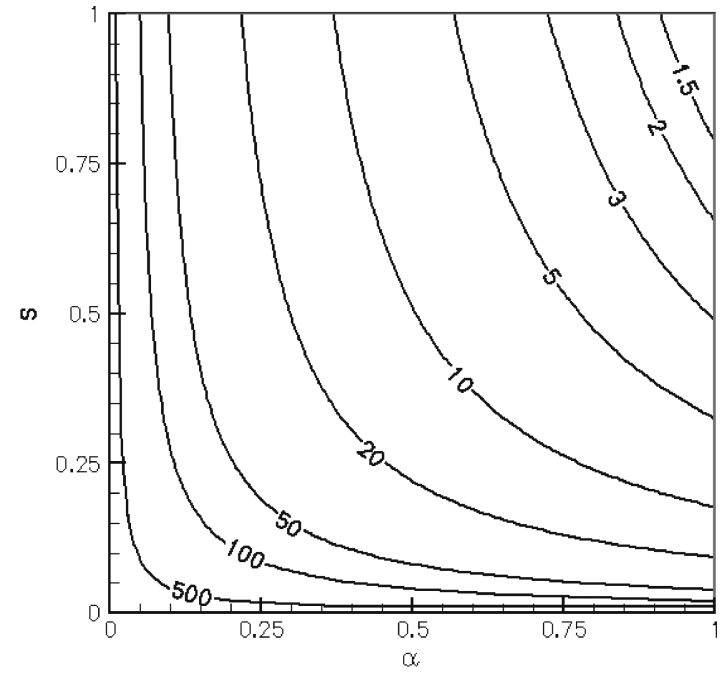

Fig. 3 Normalized backpressure contours for model $2, P_{b} S_{c}$ l $\left[\dot{m} \sqrt{ }\left(\gamma R T_{w}\right)\right]$.

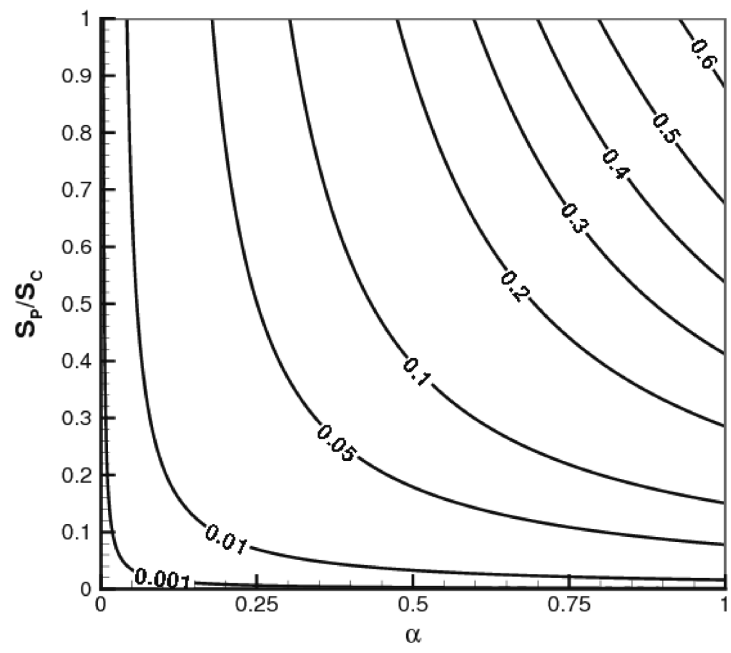

Fig. 4 Speed ratio, $U / \sqrt{ }\left(2 R T_{w}\right)$, contours predicted by model 2.

results obtained with Eq. (20a), which links the average speed ratio in the chamber with the pump sticking coefficient and the pump area. The temperature ratio set to $T_{P} / T_{w}=15 / 300$. The mean flow direction is always toward the pump, and the mean velocity increase as $\alpha$ or $s$ increases.

It is critical to consider the wall effects because many vacuum chambers are quite long and almost all particles hit the sidewall while they travel from one chamber end to the other end; hence, chamber wall effects are dominating. In vacuum chambers, there are two groups of particle diffusing back into the chamber center from the two chamber ends. The existence of a long chamber wall will impede slow particles diffusing back from the cold pump. From Eq. (3), after these slow particles reflect from a chamber wall, they resume a fast speed and the number density drops significantly. Hence, it is reasonable to conclude that the existence of a long chamber wall confines the pump effect to a small region close to the pump. Based on this observation, it may be proper to consider the effects of the chamber wall by setting the ratio $T_{p} / T_{w}$ in Eqs. (19a) and (20a) to 1 ,

$$
\begin{gathered}
n_{A}=n_{B}=\left(\dot{m} / S_{c}\right) \sqrt{\pi /\left(2 k m T_{w}\right)}(2-s \alpha) /(\alpha s) \\
U / \sqrt{2 R T_{w}}=s \alpha /[\sqrt{\pi}(2-s \alpha)]
\end{gathered}
$$

Note that this is an approximation, and later numerical simulations are used to test its validity. A final comment is that model 2 is a constant density model and a special case of the next general model. 


\section{Model 3: Kinetic, One-Sided Pumps with Wall Effects}

In contrast with the earlier treatment of considering sidewall effects by dropping the temperature ratio, the following nonconstant density model considers sidewall effects more generally. Figure 5 shows this model.

First, from the relation of the mass flow into the chamber at chamber end $\mathrm{A}$ and that out of the chamber through the pump at chamber end $\mathrm{B}$

$$
\dot{m}=m n_{\mathrm{in}} S_{c} V_{w}=\alpha m n_{B+} S_{p} V_{w}
$$

the following relation is obtained:

$$
n_{B+}=n_{\text {in }} /(\alpha s)
$$

At chamber end $\mathrm{B}$, the following relations exist for the mass flux toward the pumps and other regions of end $\mathrm{B}$,

$$
\begin{gathered}
n_{B 1-}=(1-\alpha) s \sqrt{T_{w} / T_{P}} n_{B+}=(1 / \alpha-1) \sqrt{T_{w} / T_{p}} n_{\text {in }} \\
n_{B 2-}=(1-s) n_{B+}=(1-s) n_{\text {in }} /(\alpha s)
\end{gathered}
$$

At both ends, the incoming particles are composed of two groups: One group of particles travels from the other end without any collisions with the sidewalls, whereas the other group of particles travels from the sidewall. Denote these two transportation factors $\beta$ and $\varepsilon$. Under the further assumption that the particles close to the sidewall may drift to both ends with equal probability because of the diffuse wall reflection assumption, the following equations are obtained:

$$
\begin{gathered}
n_{B+}=\beta n_{A+}+\varepsilon n_{w} \\
n_{A+}=n_{\text {in }}+\varepsilon n_{w}+\beta n_{B 2-}+\beta n_{B 1-\sqrt{T_{p} / T_{w}}}
\end{gathered}
$$

Equations (22-25) yield the following intermediate results:

$$
\begin{aligned}
& n_{A+}=\frac{(1+\beta+\alpha s-\alpha \beta s) n_{\mathrm{in}}}{\alpha s(1+\beta)} \\
& n_{A-}=\beta n_{B 1-}+\beta n_{B 2-}+\varepsilon n_{w}= \\
& \frac{\left[\beta s(1-\alpha)(\beta+1) \sqrt{T_{w} / T_{p}}+1+\beta-\beta s-\beta^{2} s-\alpha \beta s+\alpha \beta^{2} s\right] n_{\mathrm{in}}}{\alpha s(1+\beta)}
\end{aligned}
$$

Note that the percentage parameter $\varepsilon$ is totally canceled in the final results. The complete solutions for this model involve an extra geometry parameter $\beta$, which is another percentage number completely determined by the chamber length and the chamber base radius,

$$
\begin{aligned}
& n_{A}=\frac{\left[\beta s(1-\alpha)(\beta+1) \sqrt{T_{w} / T_{p}}+2+2 \beta+\alpha s-\beta s-\beta^{2} s-2 \alpha \beta s+\alpha \beta^{2} s\right] n_{\mathrm{in}}}{\alpha s(\beta+1)} \\
& n_{B}=\frac{\left[(1-\alpha) s \sqrt{T_{w} / T_{p}}+(2-s)\right] n_{\text {in }}}{\alpha s} \\
& n_{\mathrm{av}}=\frac{n_{A}+n_{B}}{2}=\frac{\left[s(1-\alpha)(\beta+1)^{2} \sqrt{T_{w} / T_{p}}+4+4 \beta+\alpha s-s-2 \beta s-\beta^{2} s-2 \alpha \beta s+\alpha \beta^{2} s\right] n_{\mathrm{in}}}{2 \alpha s(\beta+1)} \\
& f(A)=\left\{\begin{array}{l}
\frac{2(1+\beta+\alpha s-\alpha \beta s) n_{\text {in }}}{\alpha s(\beta+1)}\left(\frac{m}{2 \pi k T_{w}}\right)^{\frac{1}{2}} \exp \left(-\frac{m}{2 k T_{w}} C^{2}\right), \quad C>0 \\
\frac{2 \beta s(1-\alpha)(\beta+1) \sqrt{T_{w} / T_{p}} n_{\text {in }}}{\alpha s(\beta+1)}\left(\frac{m}{2 \pi k T_{p}}\right)^{\frac{1}{2}} \exp \left(-\frac{m}{2 k T_{p}} C^{2}\right) \\
\quad+\frac{2\left(1+\beta-\beta s-\beta^{2} s-\alpha \beta s+\alpha \beta^{2} s\right) n_{\text {in }}}{\alpha s(\beta+1)}\left(\frac{m}{2 \pi k T_{w}}\right)^{\frac{1}{2}} \exp \left(-\frac{m}{2 k T_{w}} C^{2}\right), \quad C<0
\end{array}\right. \\
& f(B)=\left\{\begin{array}{l}
\frac{2 n_{\text {in }}}{\alpha s}\left(\frac{m}{2 \pi k T_{w}}\right)^{\frac{1}{2}} \exp \left(-\frac{m}{2 k T_{w}} C^{2}\right), \quad C>0 \\
\frac{2 s(1-\alpha) \sqrt{T_{w} / T_{p}} n_{\text {in }}}{\alpha s}\left(\frac{m}{2 \pi k T_{p}}\right)^{\frac{1}{2}} \exp \left(-\frac{m}{2 k T_{p}} C^{2}\right)+\frac{2(1-s) n_{\text {in }}}{\alpha s}\left(\frac{m}{2 \pi k T_{w}}\right)^{\frac{1}{2}} \exp \left(-\frac{m}{2 k T_{w}} C^{2}\right), \quad C<0
\end{array}\right.
\end{aligned}
$$

The normalized mean velocities, the normalized pressures corresponding to experimental measurements, and the Kundsen number are

$$
\begin{aligned}
& \frac{U(A)}{\sqrt{2 R T_{w}}}=\frac{\alpha s(1+\beta)}{\sqrt{\pi}\left[\beta s(1-\alpha)(\beta+1) \sqrt{T_{w} / T_{p}}+2+2 \beta+\alpha s-\beta s-\beta^{2} s-2 \alpha \beta s+\alpha \beta^{2} s\right]}>0 \\
& \frac{U(B)}{\sqrt{2 R T_{w}}}=\frac{\alpha s}{\sqrt{\pi}\left[2-s+(1-\alpha) s \sqrt{T_{w} / T_{p}}\right]}>0 \\
& \frac{P_{b}(A) S_{c}}{\dot{m} \sqrt{\gamma R T_{w}}}=\sqrt{\frac{\pi}{2 \gamma}}\left[\frac{\beta s(1-\alpha)(\beta+1) \sqrt{T_{w} / T_{p}}+2+2 \beta+\alpha s-\beta s-\beta^{2} s-2 \alpha \beta s+\alpha \beta^{2} s}{\alpha s(\beta+1)}\right] \\
& \frac{P_{b}(B) S_{c}}{\dot{m} \sqrt{\gamma R T_{w}}}=\sqrt{\frac{\pi}{2 \gamma}}\left[\frac{2-s+(1-\alpha) s \sqrt{T_{w} / T_{p}}}{\alpha s}\right] \\
& K n=\frac{\lambda}{\sqrt{S_{c} / \pi}}=\frac{1}{\sqrt{2 \pi} d^{2} \sqrt{S_{C}} n_{A}}=\frac{\alpha s(1+\beta) m \sqrt{R T_{w} S_{C}}}{\pi d^{2} \dot{m}\left[\beta s(1-\alpha)(\beta+1) \sqrt{T_{w} / T_{p}}+2+2 \beta+\alpha s-\beta s-\beta^{2} s-2 \alpha \beta s+\alpha \beta^{2} s\right]}
\end{aligned}
$$




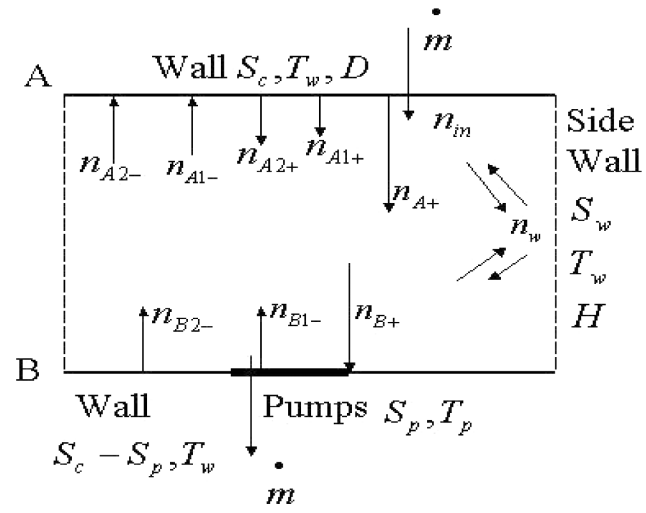

Fig. 5 Model 3.

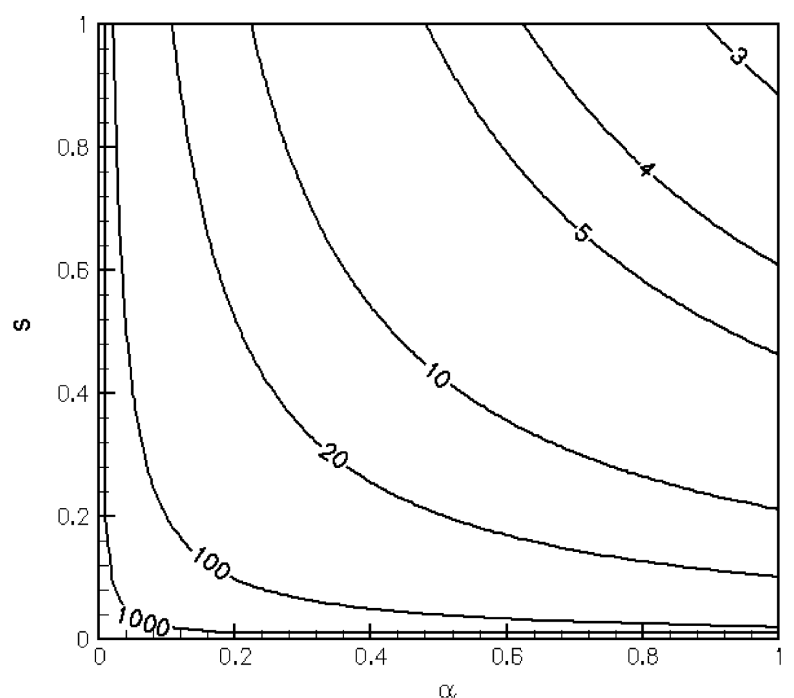

Fig. 6 Normalized backpressure contours at end A $P_{b}(A) S_{c} /$ $\left[\dot{\boldsymbol{m}} \sqrt{ }\left(\gamma R T_{w}\right)\right]$, from model $3, \boldsymbol{\beta}=\mathbf{0 . 0 5}$.

There are several significant observations from the preceding relations:

1) The formulas for chamber end B with pumps are the same as the results from model 2.

2) Generally, the number densities for A and B are different, and a nonconstant density distribution occurs. Either chamber end can have the higher density with a proper combination of parameters.

3) The background gas flows toward the pump.

4) This model can be considered as a finite chamber length model for consideration of the pump. The constant density model 2 is a special case of this model. When $\beta=1$, Eq. (28) degenerates to the constant density distribution of model 2 .

5) The highest speed ratio is the same as that of model 2 and happens at both ends with $\alpha=\beta=s=1$.

The number density in the chamber varies spatially and may be affected by various effects from the chamber, the pumps, the thruster, and the propellant. Figures 6 and 7 show results of normalized pressure and speed ratio for end $\mathrm{A}$. For both cases, the parameter $\beta$ is set to 0.05 , representing a long chamber situation. Mathematically, $\partial P / \partial s \propto\left(-s^{-2}\right)$ and $\partial P / \partial \alpha \propto\left(-\alpha^{-2}\right)$; hence, $P(A)$ is a decreasing function of $s$ and $\alpha$, and Fig. 6 shows these trends. Because the average velocity has an inverse relation with number density to maintain a fixed net mass flow rate, Fig. 7 shows an exact reverse trend in velocity when compared with density. When $\alpha$ is quite small, the model is not valid because the flow is no longer free molecular. $P_{A}$ and $P_{B}$ decrease when $\alpha$ increases, and an increase in pump area always results in a lower backpressure for both chamber ends. The velocity near the pump is always greater than zero, which means that gas flows toward the pump. The combination of $\alpha=1, \beta=1$, and $s=1$ results in $n_{A}=n_{B}=n_{\text {in }}$ and $U_{A}=U_{B}=\sqrt{ }\left(2 R T_{w} / \pi\right)$. For

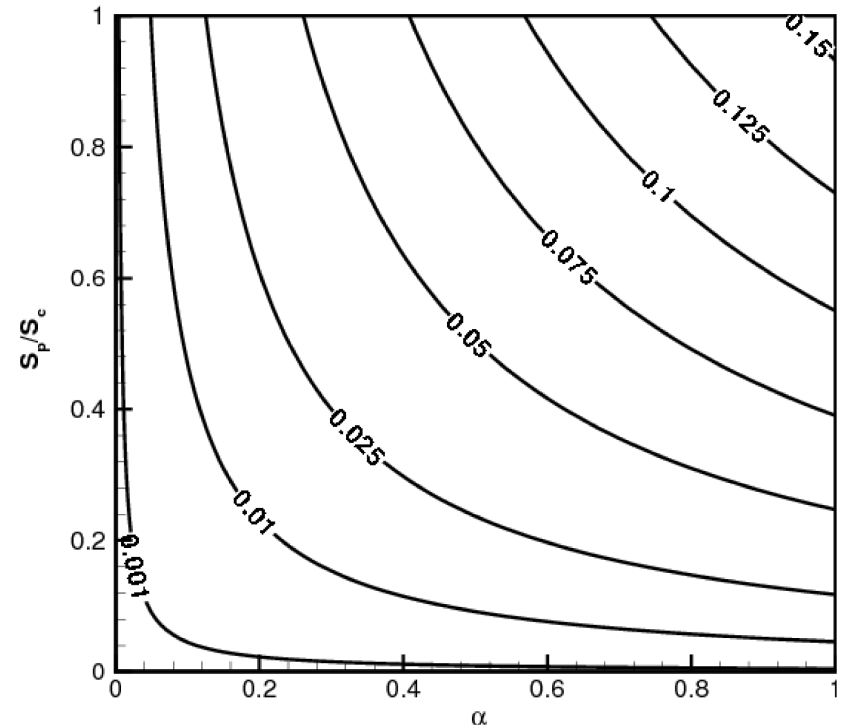

Fig. 7 Speed ratio, $U / \sqrt{ }\left(2 R T_{w}\right)$, contours at chamber end A from model $3, \beta=0.05$.

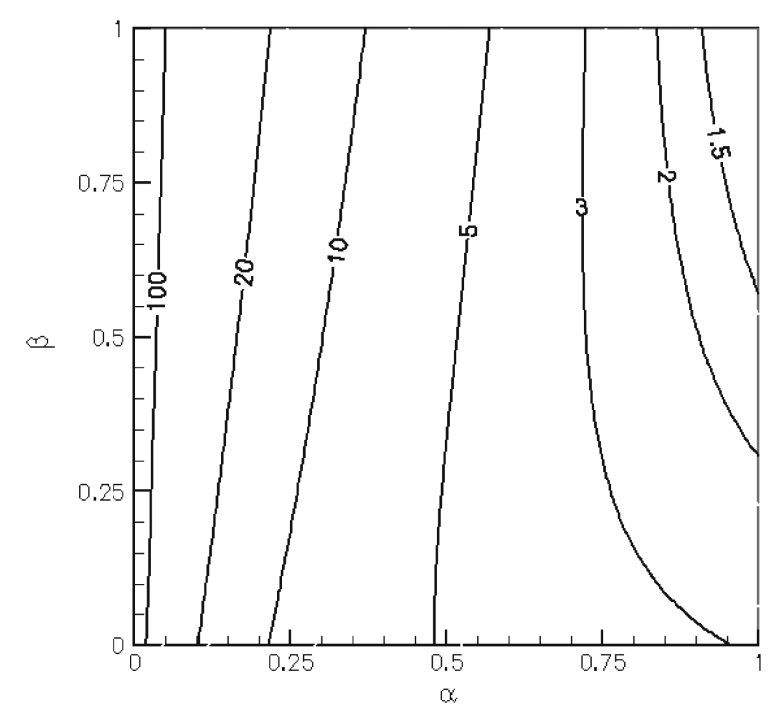

Fig. 8 Normalized backpressure contours at end A $P_{b}(A) S c /$ $\left[\dot{m} \sqrt{ }\left(\gamma R T_{w}\right)\right]$ from model $3, s=0.4$.

this situation, all particles hitting the pumps are absorbed. Because no particles are reflected from the postpump region, the number densities at chamber end $A$ and $B$ are equal to the inlet number density. For a short chamber situation $\beta=0.63$, the trends in pressure and velocity are the same as Figs. 6 and 7, though there are some difference in values.

Figures 8 and 9 show contours of normalized pressure and speed ratio for end $\mathrm{A}$ using $\alpha$ and $\beta$ as variables. For both cases, the parameter $s$ is set to 0.4. Mathematically, $\partial P / \partial \beta$ has different signs depending on $\alpha$. Hence, the wall effects have different roles in the effects of the final average stage. A small $\alpha$ results in a large amount of slow particles reflected from the pumps; within a long chamber where $\beta$ is small, these particles cannot reach the other chamber end without collisions with the chamber wall, and particle accumulations are present at both chamber end. For a short chamber where $\beta$ is greater, these particles are capable of reaching the other end; hence, a higher average density results. With a large $\alpha$, very few particles are reflected back toward the other end, and a short chamber is effective in absorbing slow particles; hence, a lower average density occurs. In Fig. 8, the condition $\partial P / \partial \beta=0$ occurs close to $\alpha=0.65$, and this result will be tested later by the numerical simulations. Average velocities shown in Fig. 9 share the same contour line shapes but reverse trends as in Fig. 8 to maintain a fixed net mass flow rate. 


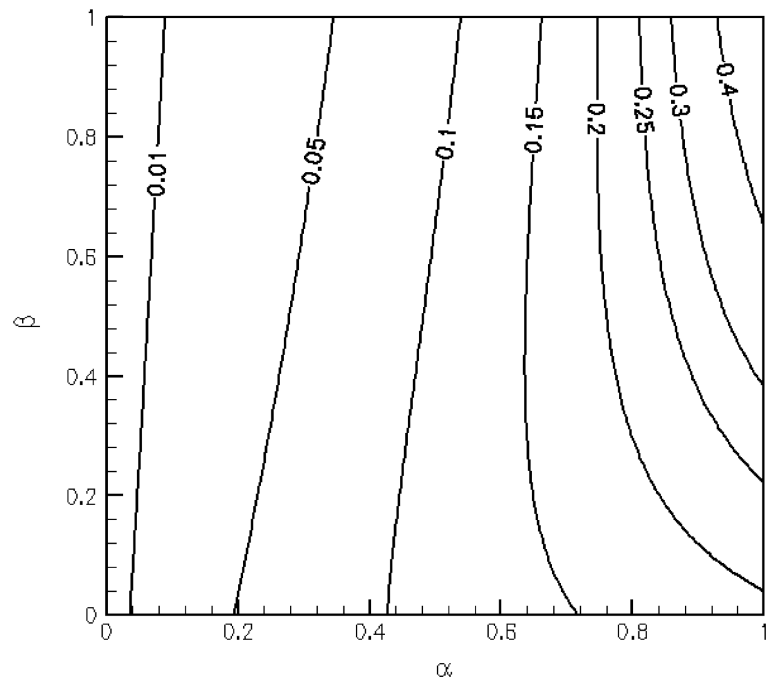

Fig. 9 Speed ratio, $U / \sqrt{ }\left(2 R T_{w}\right)$, contours at end A from model 3, $s=0.4$.

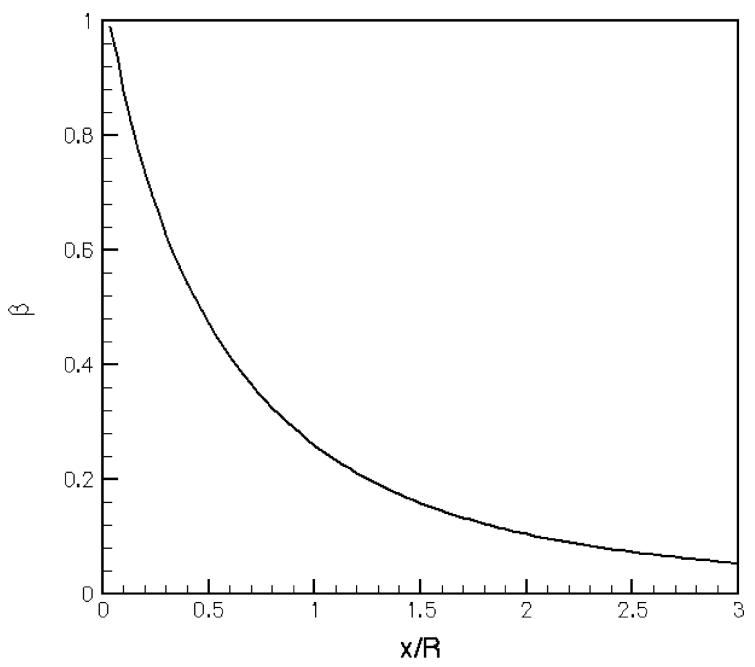

Fig. 10 Distribution of $\beta$ along chamber length.

\section{Evaluation of Transport Coefficient $\beta$}

A key parameter for this model is the transport coefficient $\beta$, which controls the probability that a particle traveling from one chamber end can reach the other chamber end without any collisions with the chamber sidewall. The evaluation of this parameter involves two steps: First, calculate the solid angles at one specific point on one chamber end subtended by the other chamber end. The solid angle formula is

$\Omega=\int_{s} \frac{\boldsymbol{r} \cdot \boldsymbol{n} \mathrm{d} S}{r^{3}}=\int_{0}^{2 \pi} H \mathrm{~d} \varphi \int_{0}^{R} \frac{\eta^{\prime 2} \mathrm{~d} \eta^{\prime}}{\left(r^{2}+H^{2}-2 r \eta^{\prime} \cos \varphi+\eta^{\prime 2}\right)^{\frac{3}{2}}}$

The final format of this integral involves the Heuman's lambda function and the complete elliptic integral of the first kind (see Refs. 12 and 13). The second step is to average the solid angles over all points on this chamber end. Though there is an analytical result for the first step, it is quite difficult to obtain an analytical expression for the second stage, which is required to evaluate the analytical results from model 3. In this study, the transportation coefficient $\beta$ is evaluated numerically. Figure 10 shows the numerical results of $\beta$ for different chamber stations at different distances from one chamber end. It shows that as $x / L$ increases, the percentage of particles reaching the planes from one chamber end drops rapidly. For a chamber with $L / R=0.9 / 3$, about $63 \%$ of the particles starting from one chamber end reach the other chamber end; for $L / R=\frac{9}{3}$, only about $5 \%$ of particles starting from one chamber end arrive at the other end without hitting the chamber sidewall. Hence, for a 9-m-long and 6-m-diam (LVTF) chamber, the sidewall effect is significant.

\section{E. Comments on Models}

Among these three models, model 1 is the crudest onedimensional model without any consideration of sidewall effects. Model 2 is a relatively accurate model in which two chamber ends and pumps and a sidewall effect is considered by setting the ratio $T_{p} / T_{w}$ to 1 . This model is actually a one-dimensional model with a constant density distribution. Model 3 is the most advanced and complete model, in which wall effects are considered, and it can predict a nonconstant density distribution along different chamber stations. Model 2 can be considered as one special case of model 3.

In experiments, it is not desirable to accumulate a large amount of particles at the chamber end equipped with pumps. Model 3 can predict a threshold value for the sticking coefficient that results in an accumulation around the chamber end equipped with pumps. For example, to satisfy $n_{A}>n_{B}$, from Eqs. (27a) and (27b), it can be shown that

$$
\alpha>\frac{\left[\beta^{2}-1+\left(1-\beta^{2}\right)\left(T_{w} / T_{p}\right)^{\frac{1}{2}}\right]}{\left[1-2 \beta+\beta^{2}+\left(1-\beta^{2}\right)\left(T_{w} / T_{p}\right)^{\frac{1}{2}}\right]}
$$

A parameter combination of $\beta=0.05$ and $T_{w} / T_{p}=300 / 15$ results in $\alpha>0.65$. Note that this relation is independent of $s$.

Another interesting factor is the temperate ratio $T_{p} / T_{w}$. Usually, the cryopumps in large vacuum chambers are maintained at low temperature, for example, the pump temperature in LVTF is approximately $12-15 \mathrm{~K}$. Generally, this temperature ratio does not have significant effects. As mentioned earlier, the large amount of slow particles reflected from the cold pumps concentrate around the pumps. Once these particles hit the chamber side wall, these particles resume a faster speed and diffuse quickly. Hence, the majority of practical long chambers is dominated with faster particles, and the pump temperature effects are not significant. Another reason accounting for the unimportance of the temperature ratio is that, in the formulas obtained in this study, the temperature ratios are usually offset by square roots and in front of these square roots there are always small coefficients. Hence, in this study, the pump temperature is set to $15 \mathrm{~K}$ and no further investigations are performed.

\section{Numerical Simulations and Discussions}

For vacuum chambers equipped with one-sided pumps, there are no experimental measurements available; hence, the validity of these models depends on comparisons with numerical simulations.

In this study, about 70 simulations using the direct simulation Monte Carlo (DSMC) method ${ }^{9}$ are performed to compare with the analytical results. A special DSMC code called MONACO ${ }^{14}$ is adopted for these simulations. In these simulations, xenon gas flows through a cylindrical chamber with a fixed base radius of $3 \mathrm{~m}$. In all of the simulations, the mass flow rates into and out of the chamber ends are set to $6.17 \mathrm{mg} / \mathrm{s}$, which is the mass flow rate out of four Hall thrusters in Ref. 2. All of the simulation results are carefully examined to confirm that the mean free path in the flowfield is over $3 \mathrm{~m}$; hence, the flow is close to free molecular for each case.

These simulations are physically accurate by including collisions, even though collisions occur very infrequently. The DSMC method simulates collision effects in rarefied gas flow by collecting groups of particles into cells. Pairs of particles inside a cell are selected at random and a collision probability is evaluated that is proportional to the product of the relative velocity and the collision cross section for each pair. The probability is compared with a random number to determine if that collision occurs. If so, some form of collision dynamics is performed to alter the properties of the colliding particles. The no time counter method ${ }^{9}$ is adopted to determine the collision rate. In this study, momentum collisions, or elastic collisions involving the exchange of momentum between the participating particles, are included in the DSMC method. The variable hard sphere ${ }^{9}$ model is employed, and the collision cross section of xenon is

$$
\sigma_{\mathrm{el}}(X e, X e)=2.12 \times 10^{-18} g^{-2 \omega} \mathrm{m}^{2}
$$




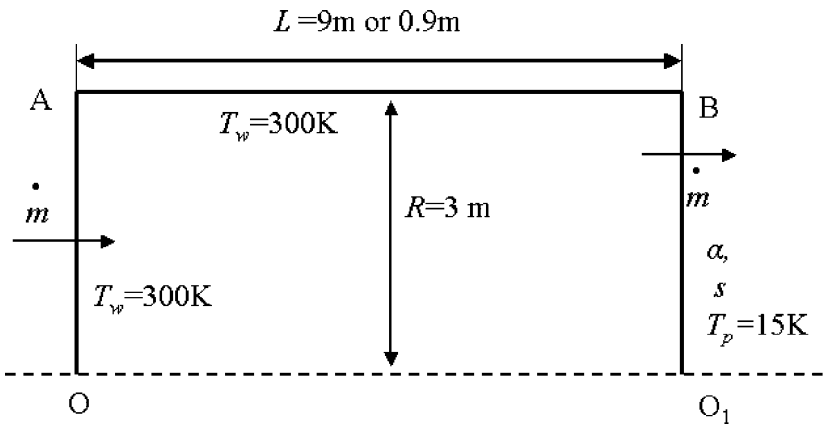

Fig. 11 Simulation domain.

where $g$ is the relative velocity and $\omega=0.12$ is related to the viscosity temperature exponent for xenon. In all elastic interactions, the collision dynamics is modeled using isotropic scattering, together with the conservation of linear momentum and energy to determine the postcollision velocities of the colliding particles.

\section{A. Boundary Conditions}

Figure 11 shows the axisymmetric simulation domain used in this study. It is a cylindrical chamber with a length of $9 \mathrm{~m}$ or $0.9 \mathrm{~m}$ and a radius of $3 \mathrm{~m}$. These simulations with a long chamber sidewall and a short chamber sidewall provide numerical results to test the performance of the analytical results obtained in this study.

There are four kinds of boundary conditions adopted in these simulations:

1) The first boundary condition is $\mathrm{OO}_{1}$, the axis of symmetry.

2) The second boundary condition is OA, a mixed inlet and diffuse wall boundary condition. When particles hit this boundary from the inner domain, they reflect diffusely with a speed characterized by a wall temperature $T_{w}=300 \mathrm{~K}$. Usually, large vacuum chambers are exposed to air; hence, a room temperature is reasonable. At the same time, this side also serves as an inlet boundary condition, and a fixed mass flow rate of $6.17 \mathrm{mg} / \mathrm{s}$ into the chamber is maintained. A uniform inlet at this side is consistent to the treatment in models 2 and 3 .

3) The third boundary condition is $\mathrm{AB}$, a diffuse wall boundary condition with $T_{w}=300 \mathrm{~K}$.

4) The fourth boundary condition is $\mathrm{BO}_{1}$, a mixed outlet and diffuse wall boundary condition. When particles hit this wall, by a probability of $1-S_{P} / S_{C}$, they reflect diffusely with a speed characterized by the wall temperature of $300 \mathrm{~K}$. By a probability of $\alpha S_{P} / S_{C}$ they are absorbed by the pumps and, hence, removed from simulation. By a probability of $(1-\alpha) S_{P} / S_{C}$ they reflect diffusely with a speed characterized by the pump temperature of $15 \mathrm{~K}$. These simulations are attempts to remove the effect of pump location; hence, there is no exact pump location are specified on this simulation domain. Whether a particle sticks to the pump or reflects on the wall or pumps is decided by comparing two random numbers and given parameters. This treatment is effective to simulate pumps of all sizes, including very small pumps.

A mesh of 45 by 15 cells along the axial and radial directions is adopted in this study. In this study, the flows are free molecular, there are very few collisions happening inside the domain, and such a mesh size provides enough resolution while minimizing the simulation cost. The time steps adopted in the final stages of these simulations vary from $1 \times 10^{-4} \mathrm{~s}$ to $1 \times 10^{-6} \mathrm{~s}$ to reduce multiple reflections for a particle in one time step. Typically, a simulation starts with quite a large time step, and a small number of particles reaches a steady state. Then the time step is reduced, and the particle number increased. For all simulations cases, approximately 1 million particles are preserved in the final sampling stage and each case takes less than $30 \mathrm{~min}$ on a Sun workstation.

\section{B. Evaluation of Average Quantities}

Models 1,2, and 3 are essentially one-dimensional models with constant distributions at all stations, and model 3 can give variations at different stations if combined with a proper interpolation of the properties at both chamber ends. The primary concern of the numerical simulations is to compute the averaged properties throughout their flowfields. At each time step, an average density and an average velocity for the whole domain are computed by counting all particles in the simulation domain and averaging all particle velocities in the domain

$$
\begin{gathered}
\rho(t)=\left[\sum\left(w_{i} N_{i}\right)\right] m / V \\
U(t)=\left(\sum U_{j}\right) / N
\end{gathered}
$$

where $N_{i}$ and $w_{i}$ are the total number of particles and particle weight in the $i$ th cell, $V$ is the whole chamber volume, and $U_{j}$ and $N$ are the $j$ th particle velocity and total number of particles in the domain. The flow evolution history can be computed through the preceding expressions.

\section{Comment About Accuracy}

The major purpose of this study is to provide several analytical expressions to evaluate the average background pressure and average background flow velocities. Because the flow is free molecular, the DSMC is an accurate simulation method to provide accurate flow solutions. When all particles in the chamber are counted and their velocities averaged, Eqs. (32a) and (32b) provide accurate average results in the whole chamber. By the inclusion of collisions, these DSMC simulations are expected to provide more accurate results than the analytical results. Hence, these particle simulation results will be used to compare the effectiveness of the analytical models. The detailed distributions in these two-dimensional simulations may be effected by subtle changes in boundary treatments, but the average number density and average velocity in the chamber are not sensitive to these changes in boundary treatment.

Though the detailed two-dimensional distributions are not the major focus of this study, observations of these results may be helpful. Figures 12 and 13 are contours of number density and velocity at a steady flow state for the case of $L / R=\frac{9}{3}, S_{p} / S_{c}=0.4$, and $\alpha=0.4$. Figure 12 shows clearly that for this case there is a large amount of particles reflected from the pump toward the other end. However, after colliding with the long chamber wall, these particles resume a faster speed and the density drops. Hence, the slow diffusion is confined to a small location around the two ends. Figure 13 shows that the velocity does not change significantly throughout the flowfield, and an overall average velocity can represent the velocity field quite well. Though there is an approximately $10 \%$ difference in the velocity distributions in the flowfield, the major focus of this study is to provide some analytical results for the average background properties. For other simulation cases, the flowfields are quite similar; but, with a higher pump area or a higher sticking coefficient,

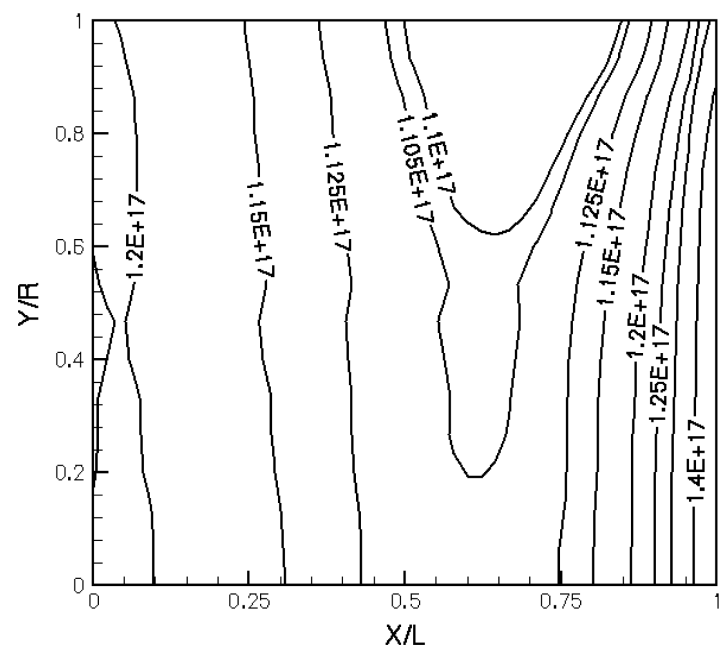

Fig. 12 Contours of number density: $L / R=\frac{9}{3}, S_{p} / S_{c}=0.4$, and $\alpha=0.4$. 


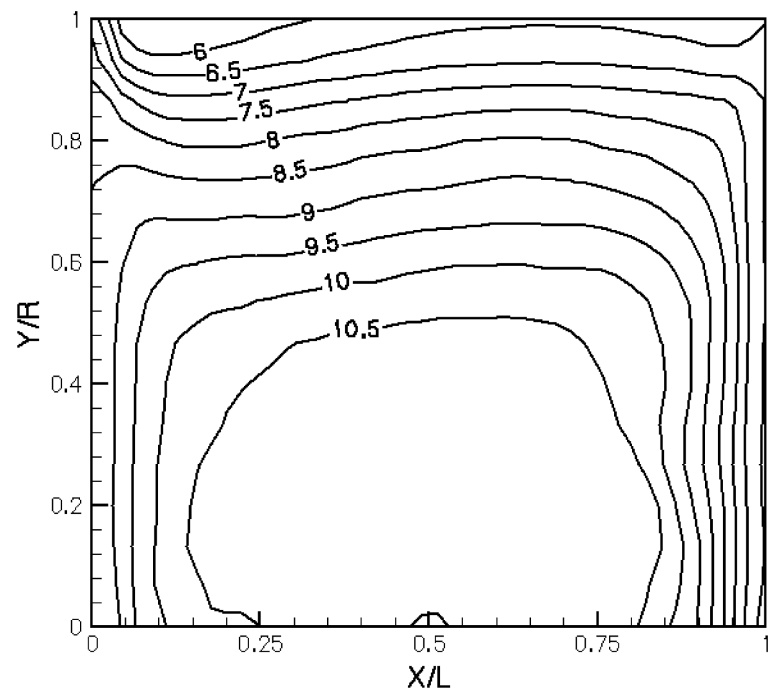

Fig. 13 Contours of velocity distribution in meters per second $L / R=\frac{9}{3}$, $S_{p} / S_{c}=0.4$, and $\alpha=0.4$.

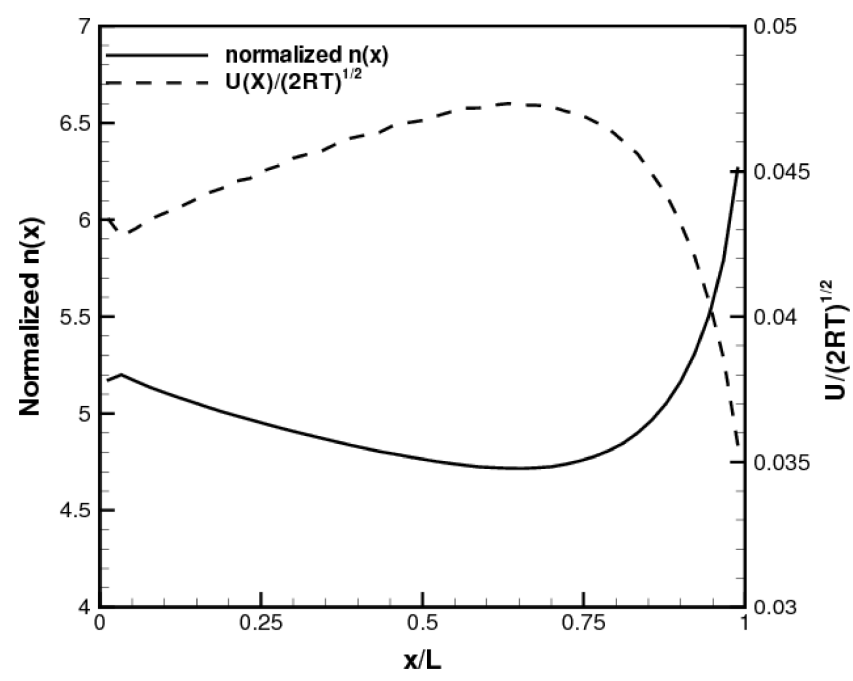

Fig. 14 One-dimensional density, normalized by $\dot{m} /\left(m S_{c}\right)$ $\sqrt{ }\left[2 \pi /\left(R T_{w}\right)\right]$, and speed ratio distribution along different stations in chamber: $L / R=\frac{9}{3}, S_{p} / S_{c}=0.4$, and $\alpha=0.4$.

the diffusion from the chamber end with pumps decreases, and, for the short chamber cases, gradients are distributed more evenly.

Note that model 3 can be used to study the one-dimensional variation of density or velocity distribution at different chamber stations. Model 3 provides two different values at the two chamber ends. With the aid of $\beta$, which is shown in Fig. 10, it is possible to construct a nonlinear distribution of density or velocity at different chamber stations. However, this will introduce a numerical distribution into analytical results, and this is not the major concern of this study. Numerically, the one-dimensional density and velocity can be calculated by averaging particles at different chamber stations. Figure 14 shows variations of steady one-dimensional density and speed results for the case $L / R=\frac{9}{3}, S_{p} / S_{c}=0.4$, and $\alpha=0.4$. For this case, the diffusion from the chamber end with pumps is more significant than from the other chamber end. This indicates that the diffusion effect is confined closely to both ends; hence, a lower value of density results for this long chamber case. The velocity varies inversely with the density to maintain a fixed net flow rate.

\section{Comparison of Numerical and Analytical Evolution History}

Model 1 provides the average number density evolution history through Eq. (5). The semidecaying period for a cylindrical chamber

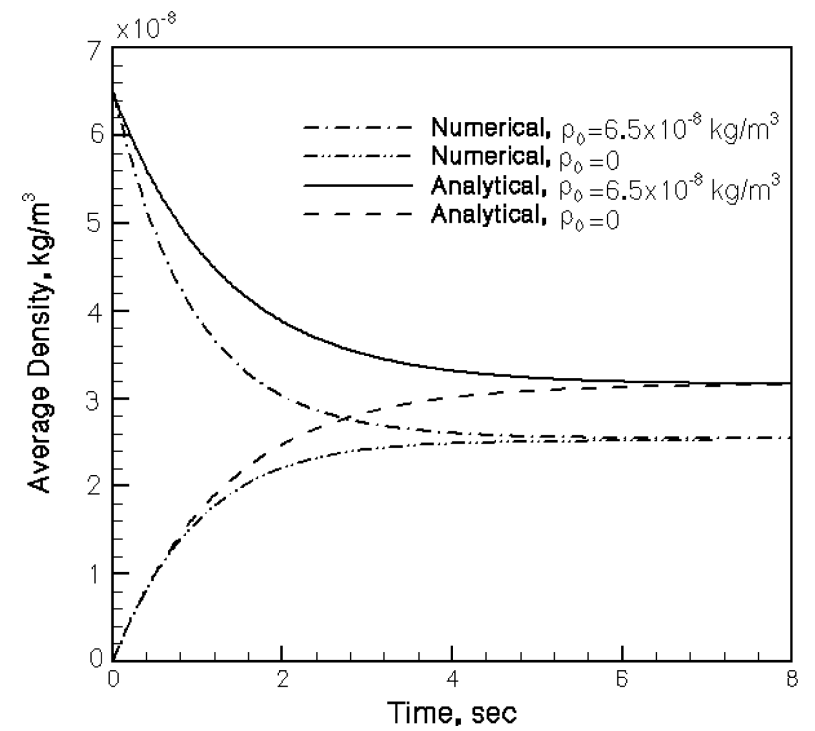

Fig. 15 Average density evolution history, $L / R=\frac{9}{3}, S_{P} / S_{C}=0.4$, and $\alpha=0.4$.

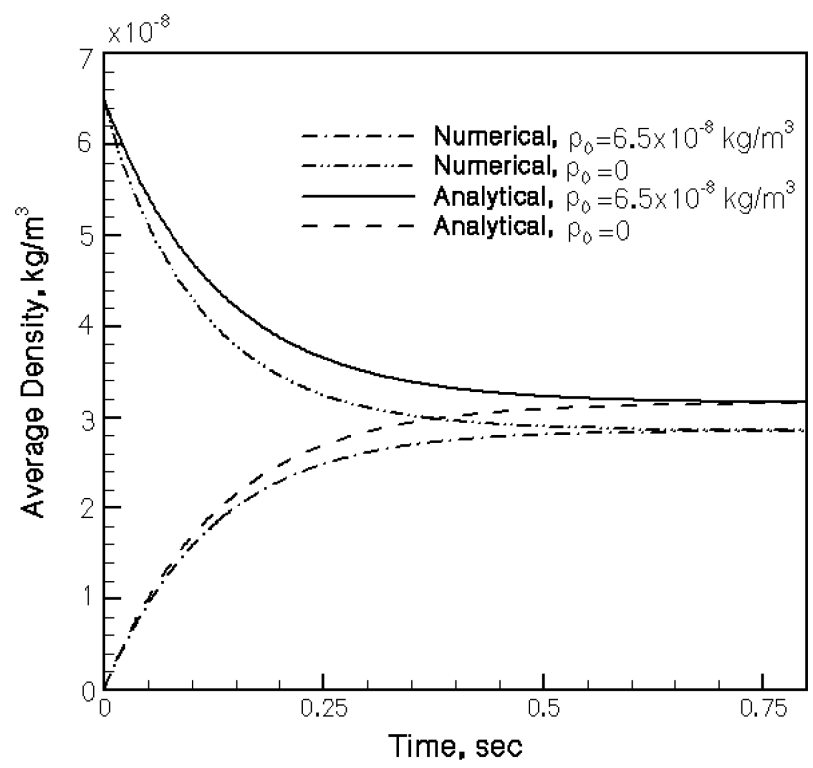

Fig. 16 Average density evolution history, $L / R=0.9 / 3, S_{P} / S_{C}=0.4$, and $\alpha=0.4$.

is

$$
\tau_{d}=\frac{V(2 \pi m)^{\frac{1}{2}}}{\alpha S_{p}\left(k T_{w}\right)^{\frac{1}{2}}}=\frac{L(2 \pi)^{\frac{1}{2}}}{\alpha s\left(R T_{w}\right)^{\frac{1}{2}}}
$$

With a xenon mass flow rate of $6.17 \mathrm{mg} / \mathrm{s}, S_{p} / S_{c}=0.4, \alpha=0.4$, and $T_{w}=300 \mathrm{~K}$. With a chamber radius of $R=3 \mathrm{~m}$ and a chamber length of $9 \mathrm{~m}$ or $0.9 \mathrm{~m}$, the semidecaying periods for the unsteady term are $\tau_{d}=1.0$ and $0.10 \mathrm{~s}$, respectively.

The density evolution process can be compared with numerical simulations. Figures 15 and 16 show two comparisons of analytical and numerical simulation results for the cases of $L / R=\frac{9}{3}$ and $L / R=0.9 / 3$, respectively. Several comments can be made based on these results:

1) The periods required to reach a steady state from the numerical simulation and analytical results are quite close. For all cases, it takes approximately four semidecaying periods to reach a steady state. There is some subtle difference between the analytical results and numerical simulation results. The analytical results assume that the backpressure is homogenous and that there is no prefered direction for gas to enter the chamber; however, in numerical simulations, at time zero, through one chamber end, 
gas begins to enter the chamber where there is already an ambient pressure or vacuum. Hence, both simulations results include a short startup time that can be estimated as by $L / \sqrt{ }\left(\gamma R T_{w}\right)=9 /$ $\sqrt{ }(5 / 3 \times 8314 / 131.25 \times 300)=0.05 \mathrm{~s}$. However, even with this extra startup time, the numerical simulations converge a little faster than theoretical results, possibly because of collisions between particles and chamber walls.

2) For situations that start with a dense initial ambient environment and a vacuum initial ambient environment, the decay times are the same.

3) The long chamber case results in a lower steady-state density for the reason mentioned earlier when discussing Figs. 8 and 14.

\section{E. Comparison of Averaged Density}

The pump sticking coefficient has an important effect on the final steady state. Figures 17 and 18 show the comparison of analytical results from models $1-3$ and 34 numerical simulations with a varying $\alpha$ and with fixed $S_{p} / S_{c}=0.4$ and 0.8 , respectively. The number density is normalized by $\left[\dot{m} /\left(m S_{c}\right)\right] \sqrt{ }[2 \pi /(R T)]$. There are two series of simulation results and two cases of analytical results from

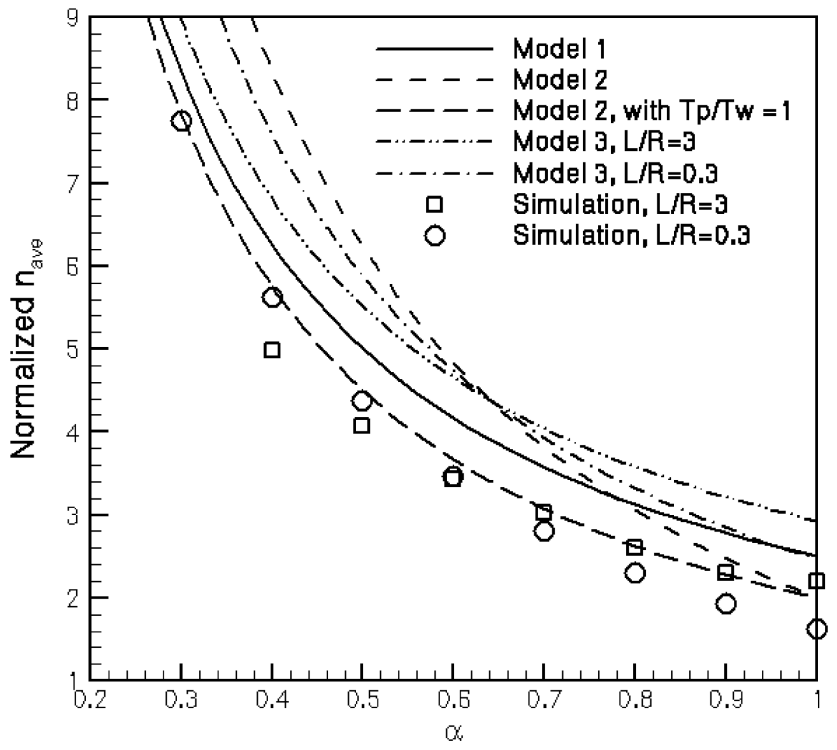

Fig. 17 Average number density inside chamber, normalized by $\dot{m} /\left(m S_{c}\right) \sqrt{ }\left[2 \pi /\left(R T_{w}\right)\right], S_{P} / S_{C}=0.4$.

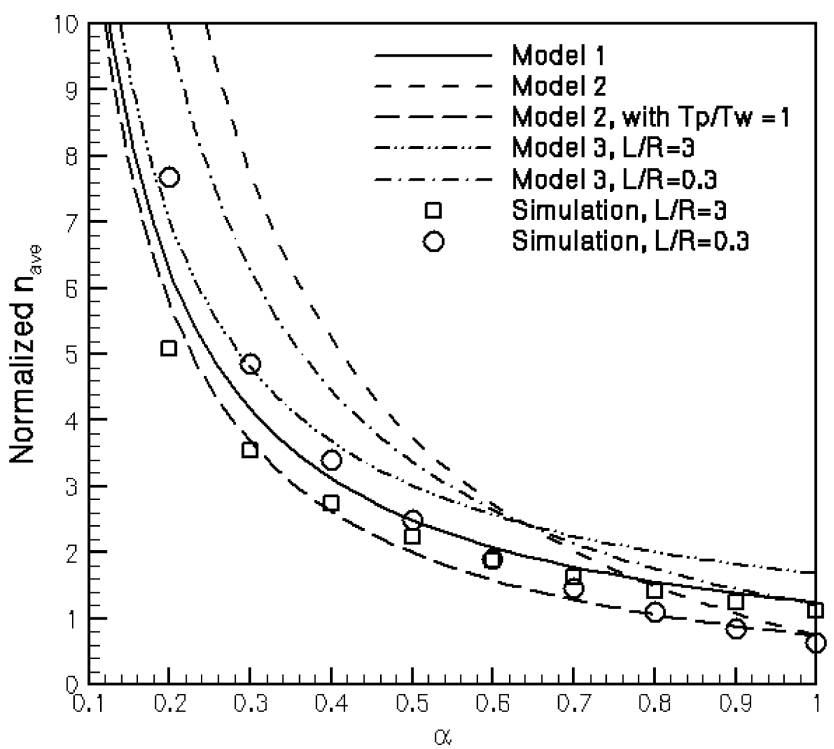

Fig. 18 Average number density inside chamber, normalized by $\dot{m} /\left(m S_{c}\right) \sqrt{ }\left[2 \pi /\left(R T_{w}\right)\right], S_{P} / S_{C}=0.8$. model 1 with $\beta=0.05$ and 0.63 , corresponding to the situations $L / R=\frac{9}{3}$ and $L / R=0.9 / 3$. In Fig. 17, the analytical result from model 1 with $\beta=0.05$ is equivalent to a horizontal intersection from Fig. 8. The simulations are divided into two groups of a long chamber wall $(L=9 \mathrm{~m})$ and a short chamber wall $(L=0.9 \mathrm{~m})$, providing two bounding lines. Because models 1 and 2 do not consider wall effects at all, and all models do not include the particle collision effects, the analytical results are less accurate than these numerical simulations. Several observations can be made from these results in Figs. 17 and 18:

1) For fixed $S_{p} / S_{c}$, the difference between analytical results decreases and the analytical results fit the simulation better as $\alpha$ increases.

2) The two series of numerical simulations yield an intersection point close to $\alpha=0.60$, which has been shown in Fig. 8 . Hence, model 3 has some superiority in predicting the trends of backpressure.

3) The $S_{p} / S_{c}=0.8$ case gives a better comparison between the numerical and analytical results than $S_{p} / S_{c}=0.4$.

4) Model 2 with $T_{p} / T_{w}=1$ gives the best performance.

The pump area has an important effect on the final steady state as well. Figures 19 and 20 show the comparison of analytical results and another 34 simulations with a variation of $S_{p} / S_{c}$ and fixed $\alpha=0.4$ and 0.8 , respectively. In Fig. 19, the analytical result $\beta=0.05$ from model 3 with $\alpha=0.4$ is equivalent to a vertical

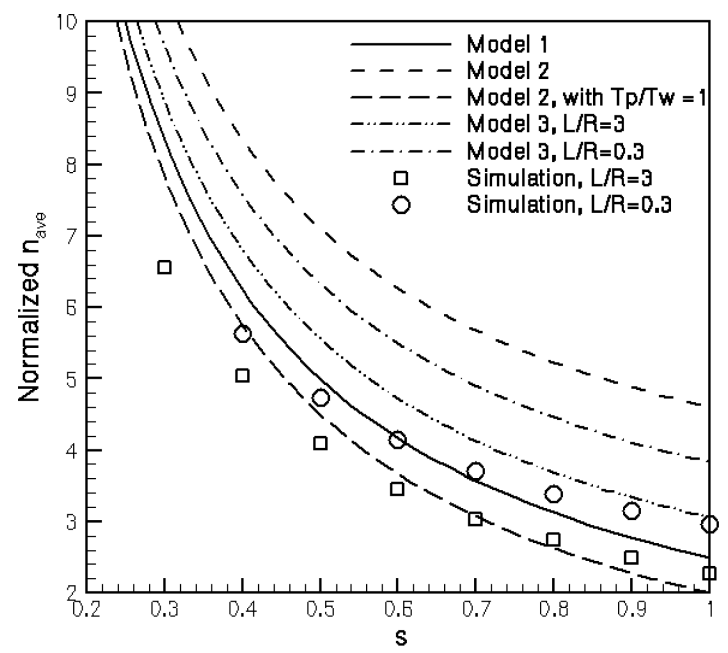

Fig. 19 Average number density inside chamber, normalized by $\dot{m} /\left(m S_{c}\right) \sqrt{ }\left[2 \pi /\left(R T_{w}\right)\right], \alpha=0.4$.

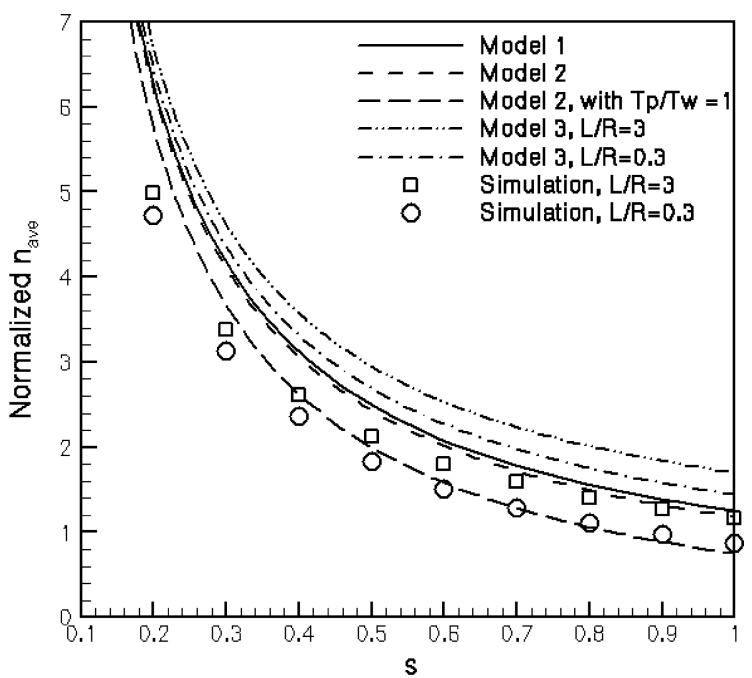

Fig. 20 Average number density inside chamber, normalized by $\dot{m} /\left(m S_{c}\right) \sqrt{ }\left[2 \pi /\left(R T_{w}\right)\right], \alpha=0.8$. 
intersection line in Fig. 8. From the results in Figs. 19 and 20, the following can be observed:

1) For fixed $\alpha$, when $s$ increases, the difference among analytical results increases, the analytical results fit the simulation better, and the difference between the two series of numerical simulation results is more pronounced.

2) The two series of numerical simulations do not have a crosspoint that is quite consistent with Fig. 8 .

3) The $S_{p} / S_{c}=0.8$ case shows better agreement between analysis and simulation than $S_{p} / S_{c}=0.4$, the difference among the analytical results decrease, and the performance of model 2 improves greatly with less particles reflected from the chamber wall.

4) The $S_{p} / S_{c}=0.8$ case is more comparable to the no-wall assumption for model 2.

5) Model 2, with a modification of $T_{p} / T_{w}=1$, has the best performance.

\section{F. Comparison of Averaged Velocity}

Figures 21 and 22 show the comparison of average speed ratios with a variation of $\alpha$ and fixed $S_{p} / S_{c}=0.4$ and 0.8 , respectively. Compared with the average number density, the agreement of average velocity results is more difficult to obtain because velocity is a higher-order momentum of the velocity distribution function. From the results in Figs. 20 and 21, the following can be observed:

1) Models 1 and 2 with $T_{p} / T_{w}=1$ have the best performance.

2) The performance of model 2 improves greatly for $S_{p} / S_{c}=0.8$.

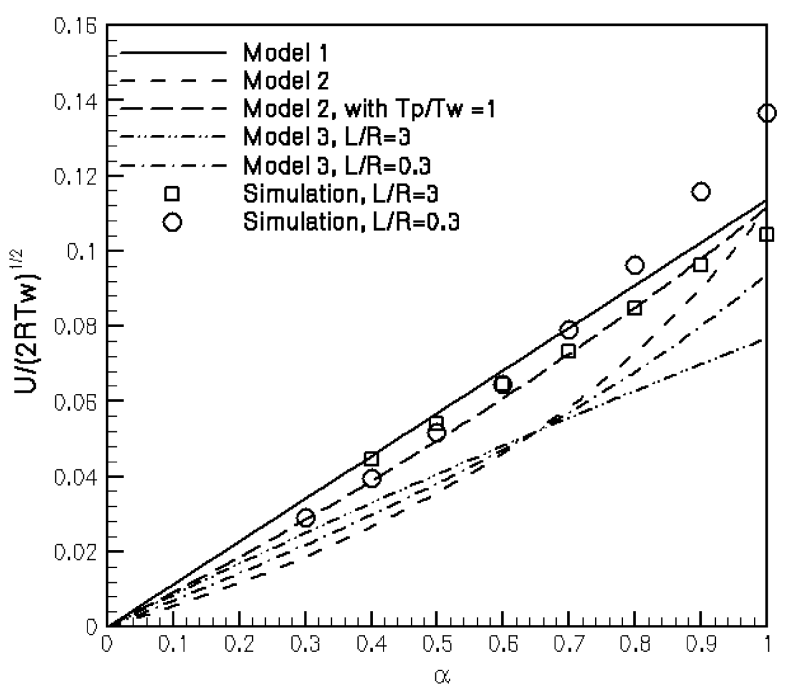

Fig. 21 Average speed ratio, $U / \sqrt{ }\left(2 R T_{w}\right)$, inside chamber, $S_{P} / S_{C}=0.4$.

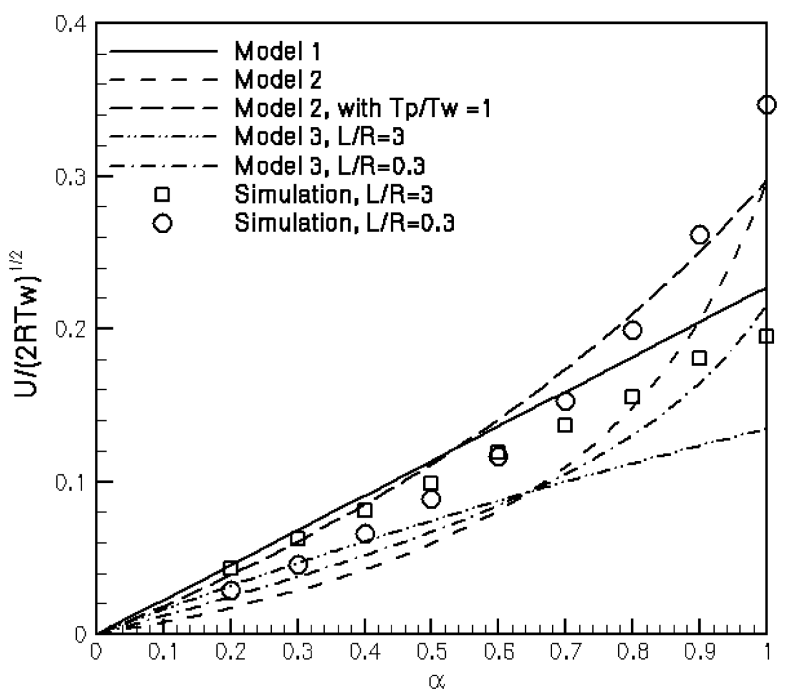

Fig. 22 Average speed ratio, $U / \sqrt{ }\left(2 R T_{w}\right)$, inside chamber, $S_{P} / S_{C}=0.8$.

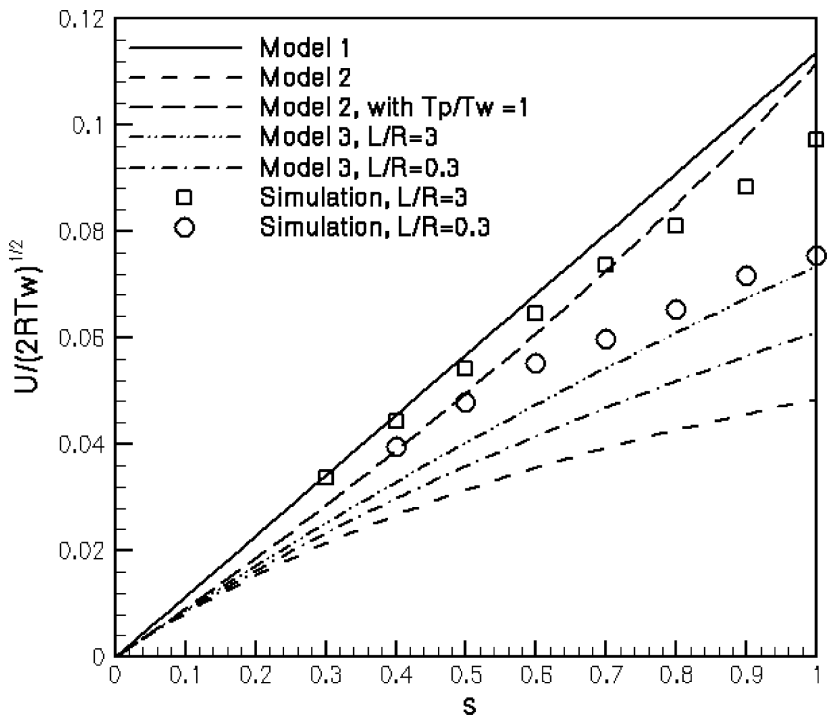

Fig. 23 Average speed ratio, $U / \sqrt{ }\left(2 R T_{w}\right)$, inside chamber, $\alpha=0.4$.

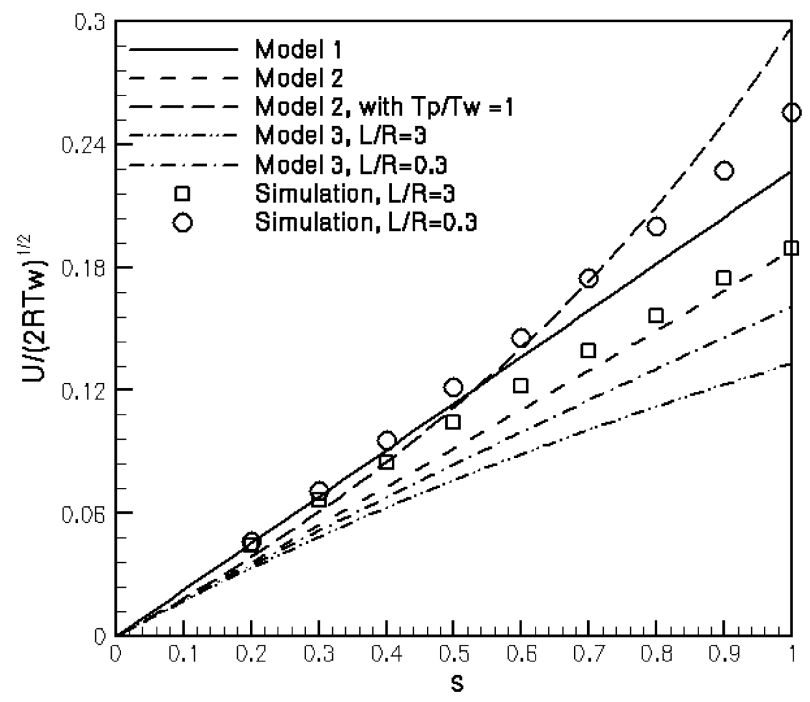

Fig. 24 Average speed ratio, $U / \sqrt{ }\left(2 R T_{w}\right)$, inside chamber, $\alpha=0.8$.

3) In Figs. 21 and 22, two series of numerical simulations intersect at $\alpha=0.6$ as indicated in Fig. 9. Again, model 3 predicts this intersection point successfully.

Figures 23 and 24 show the comparison of the average speed ratios with a variation of $S_{p} / S_{c}$ and fixed $\alpha=0.4$ and 0.8 , respectively. The following can be observed:

1) When $\alpha=0.4$, the long chamber has higher average velocities, whereas for $\alpha=0.8$ the short chamber has higher average velocities.

2) Models 1 and 2 with $T_{p} / T_{w}=1$ give the best performance.

3) Model 2 improves significantly with larger $\alpha$.

\section{Effects on Particle Simulation}

To simulate a rarefied plume flow inside a vacuum chamber, the DSMC method is usually adopted and an axisymmetric simplification substitutes in place of an expensive full-scale threedimensional simulation. Traditionally, the background flow effects are either omitted or approximated by a few static background particles in each cell. The velocities of these static background particles are randomly assigned from a zero-centered Maxwellian velocity distribution.

However, from the preceding discussions, it is demonstrated that there are essentially three groups of particles inside a vacuum chamber: two groups diffuse from the end with the vacuum pumps, whereas the last group diffuses from the other chamber end. The 
velocities for the background particles are highly affected by various factors. The plasma plume flow inside a vacuum chamber is more like firing a plume flow into another uniform background flow toward the pump. The velocity distribution for the rarefied background flow can be far from a full Maxwellian distribution, and the mean velocity of the background flow can be far from zero and can reach over $100 \mathrm{~m} / \mathrm{s}$ for xenon.

The influence on numerical simulations varies with parameters. For example, in a simulation of electric plumes firing from four Hall thrusters inside the LVTF, with a combination of parameters of $\alpha=0.39$ and $s=0.1460$ (four pumps in operation), the average background velocity inside the chamber is only $6.0 \mathrm{~m} / \mathrm{s}$, and the background number density calculated with formulas for two-sided pumps is only slightly lower than the number density calculated by $n=P_{b} /(k T)$. For this situation, the traditional approximation of background flow by using static particles with velocities sampled from a zero-centered Maxwellian distribution is quite acceptable. However, for other situations where $\alpha$ or $s$ is quite large, the background flow may have a significant effect. Hence, before performing a simplified simulation of gas flow inside a vacuum chamber, it is quite important to first evaluate the facility parameters and estimate the background flow in the chamber.

To account accurately for the rarefied background flow, there are several options:

1) Represent the backpressure by static background particles, but assign the background particles with velocities sampled from the complete analytical velocity distribution functions obtained in this study.

2) Consider the background flow with inflow boundary conditions. The front and backsides of the simulation domain use different branches of the Maxwellian distributions obtained in this study.

3) Consider the background flow with a general inflow boundary condition for all sides. This approach will require calculation of a new number density, a new mean velocity, and a new temperature from the velocity distributions obtained in this study. A new Maxwellian distribution must be constructed with the preceding information.

\section{Conclusions}

In this study, three different free molecular flow models were presented for the rarefied background flow inside a vacuum chamber equipped with one-sided pumps, and four series of numerical simulations were performed to test the validity of these analytical models.

The first model was a crude model with a strong assumption of a constant density distribution inside the vacuum chamber, and it led to a set of crude but important relations. The second and the third models were free molecular flow models considering chamber ends effects. Analyzing the flux relations and number density relations led to detailed velocity distributions for the background flow, and, based on these distributions, the mean velocities and pressures were obtained.

About 70 numerical simulations were performed to test the correctness of the analytical results. Generally, the performance of these analytical models improved greatly as $S_{p} / S_{c}$ or $\alpha$ increased. Model 2 with $T_{p} / T_{w}=1$ yielded the best performance for average density. Model 2 with $T_{p} / T_{w}=1$ or model 1 yielded the best performance in predicting the average velocities inside the chamber. Model 3 generally gave relatively poorer performance, although it is the most complete model because it involves the complex factors of $\alpha, \beta$, and $s$; it predicted the correct trend change for a long chamber and a short chamber, and it can create a nonconstant distribution between two chamber ends, which is more physically reasonable.

These models indicated that the background flow in a chamber was different from vacuum and that it cannot be simply treated as a static gas field. The highest speed reached is over $100 \mathrm{~m} / \mathrm{s}$ for xenon, and the velocity distribution function can deviate far from a Maxwellian distribution.

The formulas for the background gas obtained from this study can be used to not only evaluate or predict the performance of a vacuum chamber, but also establish a correct background flowfield for numerical simulations.

Note that in the literature, there are no previous, similar reports about how to estimate these average background properties. Meanwhile, these models and formulas are applicable to other vacuum chambers with different cross sections and different applications such as materials processing.

\section{Acknowledgments}

The work is partially supported by the U.S. Air Force Office of Scientific Research through Grant F49620-03-1-0123. The authors gratefully acknowledge the contributions to this work by Mitchell L. Walker.

\section{References}

${ }^{1}$ Walker, M. L., "Effects of Facility Backpressure on the Performance and Plume of a Hall Thruster," Ph.D. Dissertation, Aerospace Engineering Dept., Univ. of Michigan, Ann Arbor, MI, Dec. 2005.

${ }^{2}$ Beal, B. E., "Clustering of Hall Effect Thrusters for High-Power Electric Propulsion,” Ph.D. Dissertation, Aerospace Engineering Dept., Univ. of Michigan, Ann Arbor, MI, Oct. 2004.

${ }^{3}$ Hargus, W. A., and Reed, G., "The Air Force Clustered Hall Thruster Program," AIAA Paper 2002-3678, July 2002.

${ }^{4}$ Biagioni, L., Boccaletto, L., Marcuccio, S., Nicolini, D., Servi, R., and Andrenucci, M., "A Large Space Simulator for Electric Propulsion Testing: Design Requirements and Engineering Analysis," AIAA Paper 2000-3750, July 2000

${ }^{5}$ Sovey, J. S., Vetrone, R. H., Grisnik, S. P., Myers, R. M., and Parkes, J. E., "Test Facilities for High-Power Electric Propulsion," Journal of Propulsion and Power, Vol. 10, No. 1, 1994, pp. 18-25.

${ }^{6}$ Cai, C., Boyd, I. D., and Sun, Q., "Free Molecular Background Flow in a Vacuum Chamber Equipped with Two-sided Pumps," Journal of Vacuum Science and Technology, A, Vol. 24, No. 1, 2006, pp. 9-19.

${ }^{7}$ Walker, M. R., Gallimore, A. D., Boyd, I. D., and Cai, C., "Vacuum Chamber Pressure Maps of a Hall Thruster Cold-Flow Expansion," Journal of Propulsion and Power, Vol. 20, No. 6, 2004, pp. 1127-1131.

${ }^{8}$ Cai, C., "Theoretical and Numerical Studies of Plume Flows in Vacuum Chambers," Ph.D. Dissertation, Aerospace Engineering Dept., Univ. of Michigan, Ann Arbor, MI, Oct. 2005.

${ }^{9}$ Bird, G. A., Molecular Gas Dynamics and the Direct Simulation of Gas Flows, Clarendon, New York, 1991, p. 64.

${ }^{10}$ Gombosi, T., Gaskinetic Theory, Cambridge Univ. Press, New York, 1994 , p. 251

${ }^{11}$ Vincenti, W. G., and Kruger, C. H., Introduction to Physical Gas Dynamics, Wiley, New York, 1986, p. 44.

${ }^{12}$ Narasimha, R., "Orifice Flow of High Knudsen Number," Journal of Fluid Mechanics, Vol. 10, Oct. 1961, pp. 371-384.

${ }^{13}$ Narasimha, R., "Some Flow Problems in Rarefied Gas Dynamics," Ph.D. Dissertation, Dept. of Aeronautics and Physics, California Inst. of Technology, Pasadena, CA, Jan. 1961.

${ }^{14}$ Dietrich, S., and Boyd, I. D., "Scalar and Parallel Optimized Implementation of the Direct Simulation Monte Carlo Method," Journal of Computational Physics, Vol. 126, July 1996, p. 328 